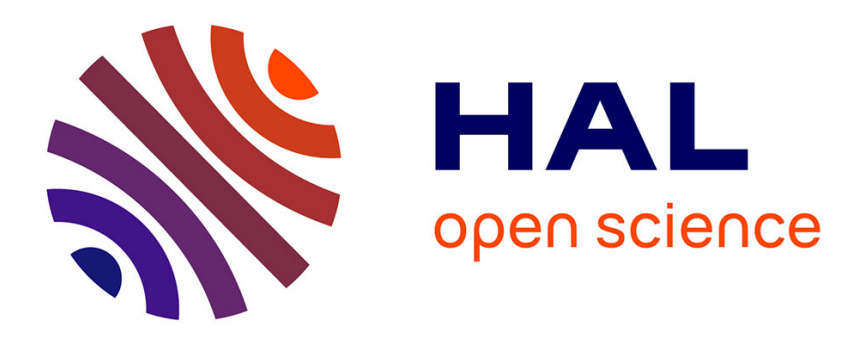

\title{
Relaxation effects in HTc-Superconducting Thin Films H. Bohn
}

\section{- To cite this version:}

H. Bohn. Relaxation effects in HTc-Superconducting Thin Films. Journal de Physique IV Proceedings, 1996, 06 (C8), pp.C8-455-C8-460. 10.1051/jp4:1996898 . jpa-00254709

\section{HAL Id: jpa-00254709 https://hal.science/jpa-00254709}

Submitted on 1 Jan 1996

HAL is a multi-disciplinary open access archive for the deposit and dissemination of scientific research documents, whether they are published or not. The documents may come from teaching and research institutions in France or abroad, or from public or private research centers.
L'archive ouverte pluridisciplinaire HAL, est destinée au dépôt et à la diffusion de documents scientifiques de niveau recherche, publiés ou non, émanant des établissements d'enseignement et de recherche français ou étrangers, des laboratoires publics ou privés. 


\title{
Relaxation effects in $H T_{c}$-Superconducting Thin Films
}

\author{
H.G. Bohn
}

\section{Forschungszentrum Jülich GmbH, Institut für Festkörperforschung, 52425 Jülich, Germany}

\begin{abstract}
Mechanical relaxation experiments have been widely applied to bulk high temperature $\left(\mathrm{HT}_{\mathrm{c}}\right)$ superconducting materials. In contrast to that only few results obtained on $\mathrm{HT}_{\mathrm{c}}$ superconducting thin films were reported. None dealt with stress driven phenomena due to the high background damping from the substrate materials used. In applied magnetic fields, however, a host reed technique can be used and the measured damping then gives information about the superconducting properties of the sample. We will discuss some experimental aspects and describe the basic information which was obtained on $\mathrm{YBa}_{2} \mathrm{Cu}_{3} \mathrm{O}_{7-\mathrm{x}}$ and $\mathrm{Bi}_{2} \mathrm{Sr}_{2} \mathrm{CaCu}_{2} \mathrm{O}_{8+\mathrm{x}}$ thin films. This includes the lower critical field, the Labusch parameter, pinning energies related to the model of thermally assisted flux flow (TAFF), and a matching effect of the flux line lattice (FLL) to the thickness of the films.
\end{abstract}

\section{Introduction}

A vast amount of experimental results has been gathered by now on the relatively new class of high temperature superconductors (HTSC) using mechanical relaxation techniques. Thè experiments can be sųbdivided into two groups: relaxation effects without (A) and with applied magnetic field (B). In the type-A experiments several internal friction peaks was found below and above the superconducting transition temperature, $T_{c}$. Some are relaxation peaks (i.e. the temperature position of the peak shifts with the measuring frequency) while others occur at fixed temperature. They are attributed to either the motion of oxygen vacancies or phase transformations, polarons, etc.. A review on these subjects is found in ref. 1. The type-B experiments deal with the properties of the magnetic flux which enters the samples if the applied field, $H_{a}$, is larger then the lower critical field $H_{c 1}$. A comprehensive review on these results has been given recently by Ziese et al. [2]. Therefore the present paper will only focus on the results specific for superconducting thin films.

\section{Experimental aspects}

While bulk HTSC both as ceramics and single crystals have been studied extensively in the past relatively few internal friction measurements on thin films have been carried out. Two materials have been used: $\mathrm{YBa}_{2} \mathrm{Cu}_{3} \mathrm{O}_{7-\mathrm{x}}(\mathrm{Y}: 123)$ and $\mathrm{Bi}_{2} \mathrm{Sr}_{2} \mathrm{CaCu}_{2} \mathrm{O}_{8+\mathrm{x}}$ (Bi:2212). Both types of films show a high degree of epitaxy (e.g. channeling experiments give minimum yields of only a few percent) when deposited on suitable 
substrates like $\mathrm{SrTiO}_{3}, \mathrm{NdGaO}_{3}, \mathrm{LaAlO}_{3}, \mathrm{KTaO}_{3}$ and $\mathrm{MgO}$. The c-axis of the perovskite structure of the films is perpendicular to the plane of the films and the $a-b$ plane is parallel to the substrate. The superconducting transition temperatures reach values of $89-95 \mathrm{~K}$ (indicating $\mathrm{x}<0.1$ ) with a transition width of $0.7 \mathrm{~K}$ and $1-10 \mathrm{~K}$ (determined from the resistive transition) for $\mathrm{Y}: 123$ and Bi:2212, respectively $[3,4]$.

The type of flux lines in the HTSC is strongly influenced by the highly anisotropic structure of the crystalline lattice (i.e. layered structures with different arrangements of the layers). The anisotropy can be quantified by the ratio $\gamma$ of the coherence lengths within the a-b planes, $\xi_{a b}$, to that perpendicular to it, $\xi_{c}$, i.e. $\gamma=\xi_{\mathrm{ab}} / \xi_{\mathrm{c}}$. For Y:123 $\gamma \approx 5$ and for $\mathrm{Bi}: 2212 \gamma \approx 150$ is found [5]. While the structure of the flux lines in Y:123 is comparable to that of conventional superconductors in Bi:2212 they consist of flux disks located in the superconducting $\mathrm{a}-\mathrm{b}$ planes.

A special feature of the HTSC is that the thermal energy may become comparable to the pinning energies of the flux lines leading to a region in the magnetic B-T phase diagram which is dominated by dynamic effects of the FLL. In particular for bulk Y:123 the FLL motion was found to be a thermally activated diffusion.

All published mechanical relaxation measurements on HTSC thin films are of type B, i.e. in applied magnetic field. The reason is the following: the total damping $Q^{-1}$ of a thin film of thickness $t_{f}$ perfectly adhering to a substrate of thickness $t_{s}$ is given by [6]

$$
Q^{-1}=Q_{s}^{-1}+\frac{3 E_{f} t_{f}}{E_{s} t_{s}} Q_{f}^{-1}
$$

where $Q_{s}^{-1}$ is the damping from the substrate and $Q_{f}^{-1}$ that from the film. $E_{f}$ and $E_{s}$ denote the corresponding Young's moduli. The prefactor of $Q_{f}^{-1}$ typically is of the order of $1 / 100$ or even smaller. Obviously one needs low damping substrate material in order to resolve the film damping (from bulk $Y: 123$ this can be estimated to be of the order of $10^{-3}$ ). Unfortunately the substrate materials which give the highest quality superconducting films show relatively high damping themselves. $\mathrm{SrTiO}_{3}$, e.g., has a ferroelectric transition near $100 \mathrm{~K}$ which results in a damping level that clearly exceeds that of the superconducting transition.

These difficulties can be overcome if one uses a host reed arrangement. Here a mechanical oscillator (e.g. a vibrating reed) is made from some low damping material like $\mathrm{Si}$ and the sample under investigation is glued to the free end of the reed. In this way no mechanical stress is introduced into the sample and any suitable substrate material can be used. This arrangement then is placed into an external magnetic field. The orientation of the sample can be varied either by gluing in different orientations to the host reed or by building a sample holder that can be rotated in situ [2]. The angle between the applied field and the plane of the film will be denoted by $\theta$. Thus $\theta=0^{\circ}$ corresponds to the magnetic field lying in the crystallographic a-b planes while $\theta=90^{\circ}$ corresponds to $H_{a}$ parallel to the c-axis.

If one uses a host reed set-up the term „mechanical relaxation“ might be misleading as the observed phenomena are clearly not driven by mechanical stress. For simplicity it may occasionally be used in the following only to indicate that the damping of a mechanical oscillation is measured.

\section{Vibrating Superconductor in a magnetic field}

The theory describing the behavior of a superconductor oscillating in an external magnetic field has been worked out by Brandt [7]. For thin film samples the width, $w_{s}$, and length, $l_{s}$, of the sample is much larger than the thickness which is typically of the order of $500 \mathrm{~nm}$ or less. Then in addition to the elastic restoring force from the host reed forces due to shielding currents and, if $H_{a}>H_{c 1}$, from the shear stiffness of the FLL occur. According to Brandt [7] both can be described by a line tension $P$

$$
P \propto H_{a}^{2} l_{s} w_{s}\left(\cos ^{2} \theta+\frac{4 t_{f}}{\pi w_{s}} \sin ^{2} \theta\right)
$$


where the first term is due to the shielding current and the second to the FLL. Obviously, as $w_{s} » t_{f}$ holds the contribution from the shielding currents dominates for thin films except for $\theta=90^{\circ}$. Since the line tension is proportional to the square of the resonance frequency this leads to an increase of the frequency in an applied magnetic field relative to that without external field.. Ziese et al. carefully measured the angular dependence of the resonance frequency and confirmed the $\cos ^{2} \theta$ dependence predicted from eq. 2 [2].

In addition the interaction between the FLL and pinning centers in the superconductor leads to an excess damping from which information about the flux line dynamics can be gained.

\section{Experimental results}

In the following paragraphs we will briefly summarize the various results which have been obtained on HTSC thin films using the vibrating reed technique.

\subsection{Labusch parameter}

Eq. 2 is strictly true for rigid pinning only. In reality, as a consequence of the sample oscillation, the flux lines move in their corresponding pinning potentials. The pinning strength can be characterized by the Labusch parameter $\alpha$ [8] which is defined as the curvature of the pinning potential. In general this parameter is difficult to measure, in particular for small samples. However, from the definition one sees that $\alpha$ is an elastic force constant which can be determined from the deviations of the frequency from the rigid pinning behavior described above. This has been done systematically as a function of temperature and applied field for a $200 \mathrm{~nm}$ thick Y:123 film [9]. E.g. at $\mu_{0} H_{a}=1 \mathrm{~T}, \theta=0^{\circ}$ and $70 \mathrm{~K}$ a value of $\alpha=4 \cdot 10^{17} \mathrm{~N} / \mathrm{m}^{4}$ is obtained which is typically $2-3$ orders of magnitude higher than for bulk Y:123. A critical discussion can be found in ref. 2 .

\subsection{Lower critical field}

As mentioned in sect. 3 not only the frequency of the oscillator is influenced by the vibration of the superconductor in an external magnetic field, but also the damping if the field is high enough to allow flux lines to enter into the sample. As pointed out by Gupta et al. [10] the simultaneous increase of the damping (due to flux line motion in the sample) and the decrease of the resonance frequency (due to a reduction of the line tension of the screening currents by the existence of vortices) constitutes a way to determine the lower critical field $H_{c \mathrm{l}}$. Hünnekes et al. applied this technique to Y:123 films with thickness varying between $43 \mathrm{~nm}$ and $600 \mathrm{~nm}$ with $H_{a}$ in the a-b planes of the films [3]. They found $\mu_{0} H_{c l}=(25 \pm 3) \mathrm{mT}$ at $20 \mathrm{~K}$ and $\mu_{0} H_{c l}=(14 \pm 3) \mathrm{mT}$ at $50 \mathrm{~K}$ independent of film thickness.

\subsection{Critical current density}

Wen et al. found for a $800 \mathrm{~nm}$ thick Y:123 film at $\theta=0^{\circ}$ that both the resonance frequency and the damping show a small hysteresis loop when the magnetic field is cycled between $0 \mathrm{~T}$ and $0.5 \mathrm{~T}$ [11]. Both the excess damping and the frequency increase are higher for decreasing field than for increasing $H_{a}$. This result was interpreted within the framework of the Bean model [12] which states that there exists a gradient of the flux density in the sample when the external field is raised or lowered. Due to pinning effects the flux is either hindered upon entering or leaving the sample interior. As the consequence the same average field distribution is obtained for two different values of the applied field the difference being $\Delta H_{a}$. From the thickness of the film and $\Delta H_{a}$ the critical current density is obtained as [11]

$$
j_{c} \approx \frac{\Delta H_{a}}{t_{f} / 2}
$$


In the temperature range between $10 \mathrm{~K}$ and $70 \mathrm{~K}$ the values obtained in this way vary between $8 \cdot 10^{9} \mathrm{~A} / \mathrm{m}^{2}$ and $4 \cdot 10^{9} \mathrm{~A} / \mathrm{m}^{2}$ in reasonable agreement with results from other experiments.

From eq. 3 one can estimate that for current densities of the order of $10^{10} \mathrm{~A} / \mathrm{m}^{2}$ and an experimental resolution of the field measurement of about $\mu_{0} \Delta H_{a}=10 \mathrm{mT}$ a hysteresis can only be observed for sufficiently thick films (typically for $t_{f}>200 \mathrm{~nm}$ ). This explains why the hysteresis effect has not been observed for other superconducting thin films.

\subsection{Relaxation effects below $T_{c}$}

If the temperature dependence of the damping is measured in applied magnetic fields an extra contribution from the moving flux lines shows up below $T_{c}$. Its magnitude depends on the strength of the field and the orientation of the sample with respect to the applied field. All published results agree that for the case of Y:123 and $\theta=0^{\circ}$ there is a strong relaxation peak slightly below $T_{c}$ while the damping remains essentially flat at lower temperatures [13-16].

The low temperature damping is proportional to $H_{a}^{2}$. It is related to flux line segments which freely move between points where they are rigidly pinned. This behavior of the flux line segments can be considered as swinging strings similar to dislocations. For rigid pinning this damping is independent of temperature in accordance with the experimental observations.

The peak just below $T_{c}$ was originally attributed to a melting transition of the FLL [13]. In the meantime most authors agree to describe this peak within the model of thermally assisted flux flow (TAFF). According to Brandt [17] the motion of the flux can be described by a diffusion equation

$$
\dot{B}=D \cdot \nabla^{2} B
$$

The activation energy for the flux diffusion is temperature and field dependent $D=D_{0} \cdot \exp (-U(B, T) / k T)$ with $U=U_{0}(B) \cdot\left(1-T / T_{\mathrm{c}}\right)^{3 / 2}[18]$, and the diffusion constant $D$ is related to a characteristic relaxation time $\tau_{0}$ by $\tau_{0}=a^{2} / D_{0}$ where $a$ is the diffusion length. It depends on the orientation of the film relative to the applied field and in the two limiting cases $\theta=0^{\circ}$ and $\theta=90^{\circ}$ essentially given by $t_{f}$ and $l_{s}$, respectively [17]. For simplicity we will follow the treatment outlined in ref. 16 and described the relaxation by a Debyetype behavior (one could equally well use the imaginary part of the a.c. susceptibility $[17,19]$ )

$$
Q^{-1}=\Delta \frac{\omega \tau}{1+(\omega \tau)^{2}}
$$

with $\tau=\tau_{0} \cdot \exp (-U(B, T) / k T)$ and $\omega / 2 \pi$ being the measuring frequency. In order to achieve a satisfactory description of the data a log-normal distribution of activation energies has to be assumed. The validity of this description can be further tested by looking at the relaxation strength $\Delta$, which in the framework of the TAFF-model is proportional to

$$
\frac{\Delta}{H_{a}^{2}} \propto w_{s}^{2} l_{s}
$$

The measured peak height is independent of the vibration amplitude, independent of the film thickness (fig. 1) and linearly dependent on the length of the sample (fig. 2) in accordance with eq. 6. There is quantitative agreement between the experimental data and the predictions from the TAFF-model which are shown in the figures as dashed lines.

Ziese et al. further point out that there is a quantitative discrepancy between the predictions from $2 \mathrm{D}$ melting theory and the results from the mechanical relaxation measurements [14]. In conclusion, the relaxation peak observed in $\mathrm{Y}: 123$ just below the transition temperature can be fully described by the TAFF-model.

Similar results have been obtained for Bi:2212 films [3]. They could also be fully explained by the TAFF model. The main difference between the two materials is that the pinning is considerably weaker in $\mathrm{B}: 2212$. This can be directly seen from the position of the relaxation peaks which occur at about $76 \mathrm{~K}$ for $\theta=0^{\circ}$ and $43 \mathrm{~K}$ for $\theta=90^{\circ}$ much lower than $T_{c}$. 


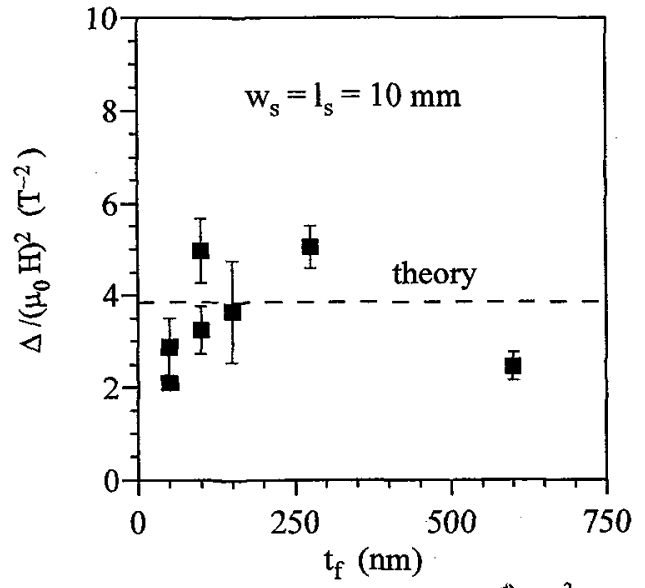

Figure 1: Reduced relaxation strength $\Delta\left(\hat{j}_{0} \mathrm{H}_{\mathrm{a}}\right)^{2}$ as a function of the film thickness. The dashed line is calculated from the TAFF model without adjustable parameter.

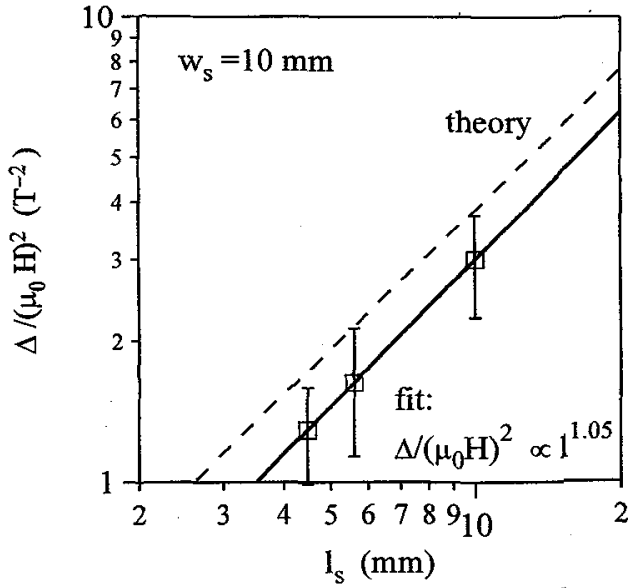

Figure 2: Reduced relaxation strength $\Delta\left(\mu_{0} \mathrm{H}_{\mathrm{a}}\right)^{2}$ as a function of the length of the sample. The dashed line (representing a linear dependence) is calculated from the TAFF model without adjustable parameter. The solid line is a fit to the data points.

\subsection{Matching effects}

If the damping of $\mathrm{Y}: 123$ films is measured at a fixed temperature $T \ll T_{c}$ as a function of the applied field and $\theta=0^{\circ}$ one finds a series of damping peaks at distinct values of the applied field [20]. The position of the peaks depends only slightly on temperature while its distance in $H_{a}$ becomes larger for thinner films. A similar behavior has been recently observed in $\mathrm{Bi}: 2212$ films [21]. Qualitatively these relaxation peaks were attributed to a matching of the FLL with the film thickness. At certain values of the applied field it is energetically more favorable to create a new row of flux lines instead of increasing the density within the existing rows. It is suggested that at this transition the flux lattice softens giving rise to large relaxation effects. These peaks, however, do not show up at all the field values where they are expected from the simple model [20]. In conjunction with the finding of similar peaks in Bi:2212 the underlying model was refined by taking into account surface barriers which have to be overcome when vortices enter or leave the film [21]. It was additionally proposed that the FLL not only has to match with the film thickness but also with the layered structure of the superconductors. The latter may give rise to selection rules which prevent the appearance of a damping peak at some of the calculated field values. However, also the improved model does not explain all the observed features in a satisfactory way.

\section{Summary}

Vibrating reed experiments have been performed on superconducting Y:123 and Bi:2212 films. Damping and frequency changes were measured in applied magnetic fields. In most cases the field was applied in the plane of the films, but also experiments were reported where the orientation of the external field with respect to the film was varied. The vibrating reed experiments have an extremely high sensitivity, they allow to study superconducting properties for films as thin as $45 \mathrm{~nm}$. In particular it is possible to measure with the external field applied in the a-b planes of the superconductor, an orientation which is not easy to access with other techniques. Among the quantities which were determined are: Labusch parameter, lower critical field, critical current density and pinning strength potentials. 
A phenomenon unique to high temperature superconducting thin films is a matching effect of the FLL to the film thickness. The occurrence of damping peaks at certain values of the applied field is tentatively ascribed to a softening of the vortex lattice when a new row of flux lines enters the sample.

\section{References}

[1] Weller M., Materials Science Forum 119-121 (1993) 667-680.

[2] Ziese M., Esquinazi P., and Braun H.F., Supercond. Sci. Technol. 7 (1994) 869-890.

[3] Hünnekes C. and Bohn H.G., J. Alloys Compd. 234 (1996) 218-224.

[4] Wagner P., Hillmer F., Frey U., Adrian H., Steinborn T., Ranno L., Elschner A., Heyvaert I., and Bruynseraede Y., Physica C 215 (1993) 123-131.

[5] Farell D.E., Rice J.P., Ginsberg D.M., and Liu J.Z., Phys. Rev. Lett. 64 (1990) 1573-1576; Martinez J.C., Brongersma S.H., Koshelev A., Ivlev B., Kes P.H., Griessen R.P., de Groot D.G., Tarnavski Z., and Menovsky A.A., Phys. Rev. Lett. 69 (1992) 2276-2279.

[6] Berry B.B. and Pritchet W.C., J. Physique 42 (1981) C5 1111-1122.

[7] Brandt E.H., Physics Lett. 113A (1985) 51-54; see also: Esquinazi P., J. Low Temp. Phys. 85 (1991) 139-232.

[8] Labusch R., Crystal Lattice Defects 1 (1969) 1-16.

[9] Ziese M., Esquinazi P., Braun H.F., and Sherman A.B., Proc. $7^{\text {th }}$ IWCC, Alpbach 1994.

[10] Gupta A., Esquinazi P., and Braun H.F., Physica B 165-166 (1990) 1443-1444.

[11] Wen Y.T., Kê T.S., Bohn H.G., Soltner H., and Schilling W., J. Phys.: Condens. Matter 4 (1992) 4519-4526. (Note: in this case the substrate was directly driven into a mechanical vibration.)

[12] Bean C.P., Phys. Rev. Lett. 8 (1962) 250-254.

[13] Gregory S., Rogers C.T., Venkatesan T., Wu X.D., Inam A., and Dutta B., Phys. Rev. Lett. 62 (1989) 1548-1551. (Note: in this case the substrate was directly driven into a mechanical vibration.)

[14] Ziese M., Esquinazi P., Gupta A., and Braun H.F, Phys. Rev. B 50 (1994) 9491-9498.

[15] Ziese M., Esquinazi P., Kopelevich Y., and Sherman A.B., Physica C 224 (1994) 79-90.

[16] Hünnekes C., Bohn H.G., Schilling W. and Schulz H., J. Alloys Compd. 211-212 (1994) 305-308.

[17] Brandt E.H., Phys. Rev. Lett. 68 (1992) 3769-3772.

[18] Malozemoff A.P., Worthington T.K., Yeshurun Y., Holzberg F., and Kes P.H., Phys. Rev. B 38 (1988) 7203-7206.

[19] Fischer K.H. and Nattermann T., Phys. Rev. B 43 (1991) 10372-10382.

[20] Hünnekes C., Bohn H.G., Schilling W., and Schulz H., Phys. Rev. Lett. 72 (1994) 2271-2273.

[21] Ziese M., Esquinazi P., Wagner P., Adrian H., Brongersma S.H., and Griessen R., Phys. Rev. B 53 (1996) 8658-8670. 